ARTICLE

https://doi.org/10.1038/s41467-019-09034-y

\title{
Bioengineered bacterial vesicles as biological nano- heaters for optoacoustic imaging
}

Vipul Gujrati1,2, Jaya Prakash², Jaber Malekzadeh-Najafabadi ${ }^{1,2}$, Andre Stiel (1) ${ }^{2}$, Uwe Klemm², Gabriele Mettenleiter ${ }^{3}$, Michaela Aichler ${ }^{3}$, Axel Walch ${ }^{3} \&$ Vasilis Ntziachristos ${ }^{1,2}$

Advances in genetic engineering have enabled the use of bacterial outer membrane vesicles (OMVs) to deliver vaccines, drugs and immunotherapy agents, as a strategy to circumvent biocompatibility and large-scale production issues associated with synthetic nanomaterials. We investigate bioengineered OMVs for contrast enhancement in optoacoustic (photoacoustic) imaging. We produce OMVs encapsulating biopolymer-melanin (OMVMel) using a bacterial strain expressing a tyrosinase transgene. Our results show that upon near-infrared light irradiation, OMVMel generates strong optoacoustic signals appropriate for imaging applications. In addition, we show that OMVMel builds up intense heat from the absorbed laser energy and mediates photothermal effects both in vitro and in vivo. Using multispectral optoacoustic tomography, we noninvasively monitor the spatio-temporal, tumour-associated OMVMel distribution in vivo. This work points to the use of bioengineered vesicles as potent alternatives to synthetic particles more commonly employed for optoacoustic imaging, with the potential to enable both image enhancement and photothermal applications.

\footnotetext{
${ }^{1}$ Chair of Biological Imaging, TranslaTUM, Technische Universität München, Munich 81675, Germany. ${ }^{2}$ Institute of Biological and Medical Imaging, Helmholtz Zentrum München, Neuherberg 85764, Germany. ${ }^{3}$ Research Unit Analytical Pathology, Helmholtz Zentrum München, Neuherberg 85764, Germany. These authors contributed equally: Vipul Gujrati, Jaya Prakash. Correspondence and requests for materials should be addressed to V.N. (email: v.ntziachristos@tum.de)
} 
broad range of synthetic nanoparticles made up of inorganic and organic materials such as quantum dots, $\mathrm{Au}, \mathrm{Ag}, \mathrm{Cu}$ and polymeric particles have been reported for diagnostic and therapeutic functionality ${ }^{1-8}$. Despite the appreciable success of synthetic nanomaterials for efficient disease diagnosis and therapy in preclinical trials, only a few synthetic agents have entered clinical trials. Factors that limit clinical dissemination of most synthetic nanomaterials include challenges involving low biocompatibility, material-associated toxicity, poor clearance and high cost of pilot scale production ${ }^{9,10}$. To overcome the technical limitations associated with synthetic nanomaterials, researchers in the last decade have shown keen interest in the development of cell-derived, nano-sized vesicles as carrier systems ${ }^{11}$. Moreover, bioengineering and bioprocessing tools have provided scalable and robust platforms for manufacturing such cells and vesicles to meet clinical and commercial needs.

Cell membrane-derived nano-vesicles have been explored with prokaryotic and eukaryotic cells, including mammalian cellderived exosomes ${ }^{12}$, erythrocyte-derived camouflaged particles ${ }^{13}$, yeast vacuoles ${ }^{14}$, bacterially derived minicells ${ }^{15}$ and outer membrane vesicles (OMVs) ${ }^{16,17}$. Exosome-based systems show low stability and yield and require expensive purification and production methods. Erythrocyte-derived particles exhibit excellent biocompatibility because they are taken from the target organism, but their lack of a nucleus means that they cannot be genetically engineered to carry biologically derived cargo. Yeast vacuoles show good ability to penetrate tissues and are easy to scale up, but their long-term stability, immunogenicity and toxicity remain to be clarified. Bacterial OMVs show several advantages as nanocarriers since they attain a rigid membrane, which imparts stability and reduces leakage in systemic circulation. Moreover, OMVs are safe because they are acellular and can be used in vivo in very small quantities. Importantly, OMVs can be customised to carry desired payloads and can easily be produced in large quantities using fermentation and purification procedures previously optimised on a pilot scale. This is particularly beneficial when considering that bacteria can easily be modified genetically to produce desired agents useful in vaccination, bio-sensing, bioimaging, therapy or targeted delivery; and these agents can be localised specifically in membrane-derived vesicles ${ }^{16-20}$.

In this work we consider OMVs a platform for optoacoustic applications, which can be employed for contrast-enhancement and therapeutic applications. Multi-spectral optoacoustic tomography (MSOT) is a non-invasive imaging technique that illuminates tissue at many near-infrared (NIR) wavelengths and performs spectral detection of endogenous chromophores based on the absorption spectrum. The method has been shown capable to detect melanin, oxygenated and deoxygenated haemoglobin and lipids or externally administered photo-absorbing synthetic probes, within depths of several millimetres to centimetres ${ }^{21-24}$. A major challenge for implementing MSOT in basic research and clinical procedures is identifying moieties that enable contrast enhancement for improving detection of particular pathophysiological conditions. Melanin is found naturally in many living organisms and it absorbs strongly in the visible and NIR window $^{25}$. Optimised as a natural absorber of light, melanin is therefore well suited for enhancing the contrast for optoacoustic imaging 26,27 . In addition, melanin has high photothermal conversion efficiency and is consequently highly suitable for photothermal therapy, i.e., treatment whereby light illumination of tissue that selectively contains melanin induces local heating that kills tumour cells ${ }^{28}$. The ability of melanin to serve both as a contrast enhancement and a therapeutic agent makes it appropriate for theranostics, i.e., the combination of diagnostics and therapeutics in a single agent. In that respect, melanin could not only provide contrast and improve the detection abilities for optoacoustic imaging, but also enable local therapy which can be monitored by optoacoustic imaging ${ }^{29}$. Harnessing the contrast enhancement and theranostic potential of melanin, however, requires overcoming its low solubility, which necessitates treatment with alkaline solvents and conjugation with hydrophilic polymers ${ }^{27,28}$. Such processing steps are costly and make scale up challenging. Therefore, developing a biological approach to package melanin inside cell membrane-derived nanocarriers would be of great benefit for diagnosis and therapy.

We hypothesise that we can package naturally occurring melanin into bacterial OMVs to create a biocompatible nanomaterial $\left(\mathrm{OMV}^{\mathrm{Mel}}\right)$ for efficiently delivering the photoabsorber to target tissues for optoacoustic imaging and theranostic applications. In order to avoid systemic side effects due to bacterial endotoxin lipopolysaccharide (LPS), we use an Escherichia coli strain previously modified to be less endotoxic through inactivation of the $m s b B$ gene (to give $\left.\mathrm{OMV}^{\Delta \mathrm{msbB}}\right)^{16}$, and we further engineer it to overexpress tyrosinase, which produces melanin that is passively incorporated into the cytosol and membrane of $\mathrm{OMV}^{\Delta \mathrm{msbB}}$ (to give OMV $\left.{ }^{\mathrm{Mel}}\right)$. We test the ability of MSOT to detect OMVMel in phantoms and in vivo, and the vesicles generate strong MSOT signals in both cases. Systemically administered OMVs passively target and accumulate in tumour tissue via the enhanced permeability and retention (EPR) effect. We examine the ability of $\mathrm{OMV}^{\mathrm{Mel}}$ to produce local heating in vitro after irradiation with a pulsed NIR light source, and we confirm that this local heating retards tumour growth in vivo (photothermal treatment). Furthermore, we show that a single dose of $\mathrm{OMV}^{\mathrm{Mel}}$ inhibits tumour growth while triggering only mild, short-term systemic inflammation. These results establish $\mathrm{OMV}^{\mathrm{Mel}}$ as a promising agent for optoacoustic imaging and potentially theranostics, even suggesting that OMVs may be able to inhibit tumour growth through synergy of photothermal effects and cytokine-mediated antitumour responses ${ }^{17}$. In future, it may be possible to replace the melanin with other naturally derived theranostic cargos to generate a flexible platform for imaging-based theranostic applications against cancer and other diseases.

\section{Results}

OMV isolation, purification, and characterisation. Figure 1 illustrates our approach to produce $\mathrm{OMV}^{\mathrm{Mel}}$. We started with a strain of E. coli K12 (derived from W3110) carrying an inactivated $m s b B$ gene, which leads to underacetylated and therefore much less endotoxic lipid A, an integral component of LPS $16,30,31$. We further engineered the bacteria to express Rhizobium etli tyrosinase $^{32}$, which is the rate-limiting enzyme in melanin biosynthesis. The idea was that melanin would be produced and would accumulate in the cytosol and periplasmic space, then it would be packaged into membrane and cytosol of OMVs that would be shed into the culture medium. OMVs were purified from bacteria carrying the tyrosinase gene (OMV $\left.{ }^{\mathrm{Mel}}\right)$, from the parental bacteria with an inactivated $m s b B$ gene $\left(\mathrm{OMV}^{\Delta \mathrm{msbB}}\right)$, and from wild-type bacteria $\left(\mathrm{OMV}^{\mathrm{WT}}\right)$ through multiple centrifugation steps. Figure $2 \mathrm{a}$ shows that the buffer containing isolated OMVMel appeared black, whereas OMVWT appeared light brown. Figure 2b, c shows OMV characterisation in terms of size and morphology. As evident from hydrodynamic size and transmission electron microscope analysis, most vesicles were in the range of $20-100 \mathrm{~nm}$ and had a uniform circular, bilayer morphology. Their sub-micron size makes the OMVs suitable for in vivo optoacoustic imaging of tumours. The colour difference between wild-type and melanin-containing vesicles was evident in transmission electron micrographs. OMVWT was monitored after negative staining, whereas OMV ${ }^{\mathrm{Mel}}$ was visible without staining. 


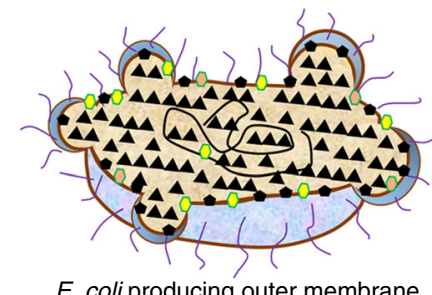

E. coli producing outer membrane
vesicles containing melanin (OMVMel)

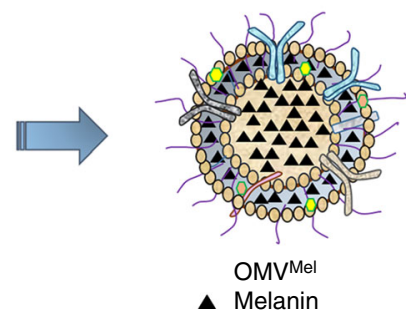

Fig. 1 Schematic representation of OMVMel generation. A schematic representation of $\mathrm{OMVMel}$ purified after vesiculation from the parental bacteria. OMV, outer membrane vesicle

We tested whether the concentrated melanin inside $\mathrm{OMV}^{\mathrm{Mel}}$ could give a strong MSOT signal. Figure $2 \mathrm{~d}$ shows that phantoms of $\mathrm{OMV}^{\mathrm{Mel}}$ gave $\sim 7$-fold stronger optoacoustic signal than phantoms of $\mathrm{OMV}^{\mathrm{WT}}$ after illumination at $750 \mathrm{~nm}$. The absorption spectrum of $\mathrm{OMV}^{\mathrm{Mel}}$ resembled that of pure melanin $^{33}$, consistent with the idea that $\mathrm{OMV}^{\mathrm{Mel}}$ contains encapsulated melanin. These results suggest that OMVMel can be used as an MSOT imaging probe, such as for basic studies of tumour biology, diagnostic studies and longitudinal monitoring of treatment response.

Photothermal heating efficiency and cytotoxicity of $\mathrm{OMV}^{\mathrm{Mel}}$. Melanin shows promise not only for disease research and diagnosis, but also for treatment. Irradiating the biopolymer can trigger local heating that kills diseased tissue through photothermal ablation or photothermal therapy ${ }^{28,34}$. We therefore asked whether the melanin packaged within $\mathrm{OMV}^{\mathrm{Mel}}$ shows potential to act as a nanoheater for photothermal therapy. Figure $3 \mathrm{a}$ shows that irradiating a suspension of OMVMel led to much greater heating than irradiating OMVWT or phosphatebuffered saline (PBS), and that the rate of temperature increase was dependent on the concentration of melanin-carrying vesicles. Figure $3 \mathrm{~b}$ shows that $\mathrm{OMV}^{\mathrm{Mel}}$ solutions increased by $>10^{\circ} \mathrm{C}$ from room temperature after only $2 \mathrm{~min}$ of laser irradiation, and that this heating effect persisted. This performance is promising, given preclinical studies showing that heating cancer cells to $42-50{ }^{\circ} \mathrm{C}$ for more than 5 min can be lethal ${ }^{35-37}$. Figure $3 \mathrm{c}$ shows that the experimentally determined absorption coefficient of $\mathrm{OMV}^{\mathrm{Mel}}$ was $0.75 \mathrm{~cm}^{-1}$ (absorbance of $0.31 \mathrm{OD}$ ) at $750 \mathrm{~nm}$. This is an order of magnitude greater than the absorption coefficient of tissue (approximately $0.1 \mathrm{~cm}^{-1}$ ) ${ }^{33}$, suggesting that photothermal effects can be induced selectively where $\mathrm{OMV}^{\mathrm{Mel}}$ deposits and not in neighbouring tissue. Figure $3 \mathrm{~d}$ shows that $\mathrm{OMV}^{\mathrm{Mel}}$ exhibits photothermal conversion efficiency of $18.65 \%$ with a quantity of $\mathrm{OMV}^{\mathrm{Mel}}$ equivalent to approximately $150 \mu \mathrm{g}$ of $\mathrm{OMV}^{\mathrm{WT}}$ (see Methods); this efficiency depends on melanin's concentration and molar absorptivity $1,2,38,39$. To test the potential for photothermal therapy in living cells, we exposed $4 \mathrm{~T} 1$ breast cancer cultures to $\mathrm{OMV}^{\mathrm{Mel}}$, then irradiated the treated cells for several minutes with a tuneable nanosecond-pulsed laser operating from $730 \mathrm{~nm}$ to $830 \mathrm{~nm}$. The total laser operating time was $6 \mathrm{~min}$, which included both wavelength tuning and irradiation time. Figure $3 \mathrm{e}$ shows that the number of apoptotic cells, which appeared red because of their ability to bind ethidium homodimer-1, was much greater in cultures treated with $\mathrm{OMV}^{\mathrm{Mel}}$ than in cultures untreated or treated with $\mathrm{OMV}^{\mathrm{WT}}$.

In vivo optoacoustic imaging of $\mathrm{OMV}^{\mathrm{Mel}}$. As a first step towards validating the ability of $\mathrm{OMV}^{\mathrm{Mel}}$ to accumulate in tumours in vivo, we injected mice carrying subcutaneous $4 \mathrm{~T} 1$ mouse mammary gland tumours with $\mathrm{PBS}, \mathrm{OMV}^{\mathrm{WT}}$ or $\mathrm{OMV}^{\mathrm{Mel}}$ via the tail vein. Figure $4 \mathrm{a}$ shows time-dependent accumulation of $\mathrm{OMV}^{\mathrm{Mel}}$ in tumour tissue using optoacoustic imaging. At $3 \mathrm{~h}$ after injection, unmixed melanin signal was much higher in animals treated with $\mathrm{OMV}^{\mathrm{Mel}}$ than in other animals. This strong optoacoustic signal was evident even at $24 \mathrm{~h}$ after injection, suggesting persistence in the circulation and, potentially, accumulation in tumours. Next, we defined regions of interest within tumours and quantified levels of melanin present. Figure $4 \mathrm{~b}$ clearly indicates accumulation of $\mathrm{OMV}^{\mathrm{Mel}}$ in tumours via the EPR effect. Melanin signal was higher at $3 \mathrm{~h}$ than at $24 \mathrm{~h}$ after injection, indicating that some particles may undergo systemic clearance via the tumour vasculature. Given the overlap in absorption spectra between melanin and deoxyhaemoglobin, we wanted to confirm the presence of melanin based on spectral analysis. The spectral signature of deoxyhaemoglobin and melanin show lower absorption with increasing wavelength and are not orthogonal to each other. Thus, identification of melanin in tumours is a major challenge, in large part because tumours tends to be hypoxic relative to normal tissue, and hence deoxyhaemoglobin concentration is relatively high. Figure $4 \mathrm{c}$, d compares the absorption spectra in the regions of interest in tumours from animals treated with PBS or OMV ${ }^{\mathrm{Mel}}$. As wavelength increased from 800 to $900 \mathrm{~nm}$, optoacoustic signal decreased sharply (indicated with a black line) in the OMV ${ }^{M e l}$ group but remained constant in the PBS group. These results are consistent with the idea that the spectra obtained from OMVMel_ treated tumours are a combination of deoxyhaemoglobin and melanin spectra, whereas the spectra from PBS-treated controls reflect primarily deoxyhaemoglobin, for which the optoacoustic signal has been shown to remain constant between 800 and $900 \mathrm{~nm}^{33}$. In addition, the biodistribution of OMV $\mathrm{OMel}^{\mathrm{M}}$ in a mouse was monitored during $120 \mathrm{~min}$ after intravenous injection (we did not examine non-tumour biodistribution over longer periods because we had to ensure that the animal did not move between measurements, and ethical considerations limited how long we could maintain the animal anaesthetised inside the MSOT setup). During this $2 \mathrm{~h}$ period, the MSOT signal gradually increased in the tumour, tumour-adjacent region just below the skin as well as in liver and kidney (Supplementary Figure 1a). This provides further evidence that melanin is the primary source of contrast in our MSOT set-up, and it suggests that OMVMel circulates and distributes in various organs. At the same time, the melanin signal in tumours rose consistently even from early time points (Supplementary Figure 1b) and our experiments at 3 and $24 \mathrm{~h}$ (Fig. 4a) showed appreciable signal in tumours. This persistent signal in the tumour suggests passive targeting ability of $\mathrm{OMV}^{\mathrm{Mel}}$, likely due to nanometre size that ensures penetration through the leaky blood vessels and due to EPR effects in the tumour region.

In vivo photothermal therapy. To assess the potential of $\mathrm{OMV}^{\mathrm{Mel}}$ for cancer treatment, we conducted photothermal experiments on nude mice bearing 4T1 tumours. Animals were randomly allocated to receive PBS intravenously or to receive $\mathrm{OMV}^{\mathrm{WT}}$ or $\mathrm{OMV}^{\mathrm{Mel}}$ intravenously or intratumourally. At $3 \mathrm{~h}$ after administration, animals were exposed (or not) to a continuous wave laser $\left(1.5 \mathrm{~W} \mathrm{~cm}^{-2}, 800 \mathrm{~nm}\right)$ for $6 \mathrm{~min}$. Tumour growth rate and animal body weight were recorded at fixed intervals. As can be seen in Fig. 5a, the tumour surface temperature in the laser-exposed area in animals treated intratumourally with $\mathrm{OMV}^{\mathrm{Mel}}$ reached as high as $56^{\circ} \mathrm{C}$, compared to $47^{\circ} \mathrm{C}$ in animals treated intravenously with $\mathrm{OMV}^{\mathrm{Mel}}$ and $39^{\circ} \mathrm{C}$ with PBS. This likely reflects much more efficient delivery of $\mathrm{OMV}^{\mathrm{Mel}}$ to tumours when they are injected directly. The much greater heating observed with intratumoural administration of $\mathrm{OMV}^{\mathrm{Mel}}$ was associated with superior therapeutic effect: in 
a

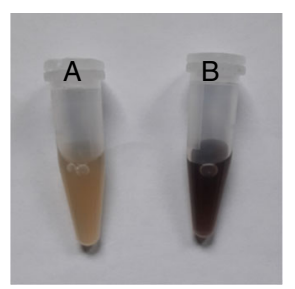
A: OMVWT
B: OMVMel

C

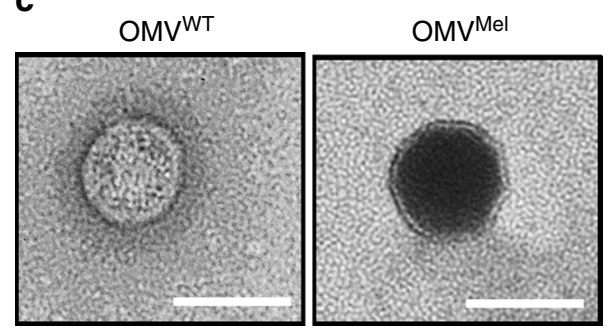

b
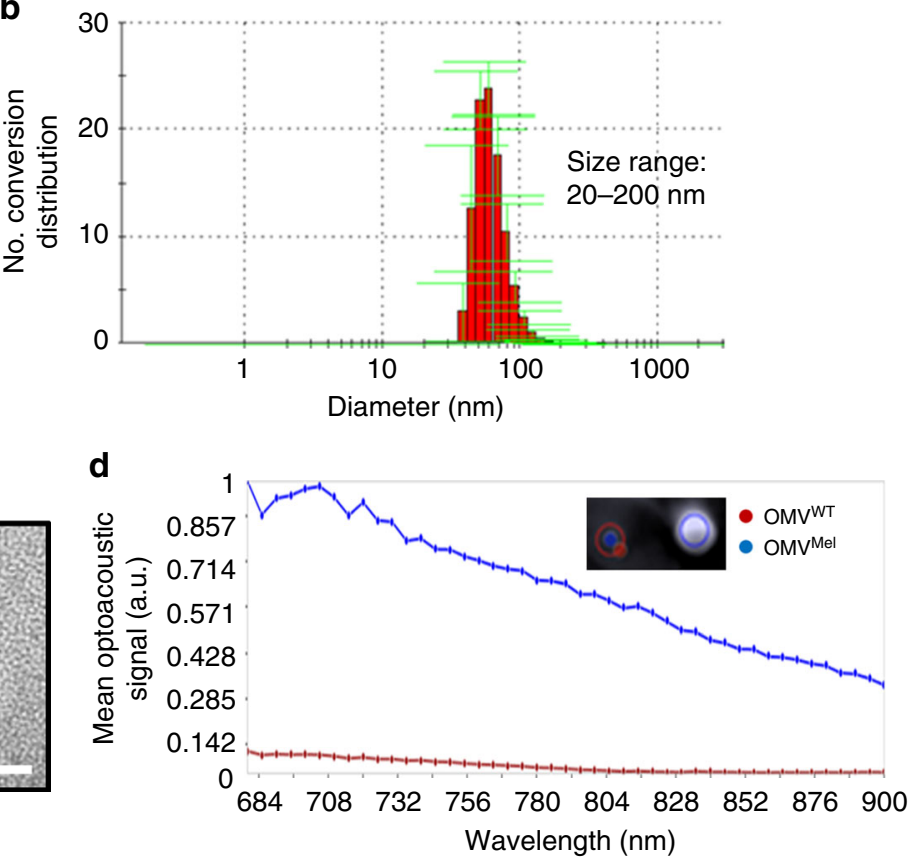

Fig. 2 Outer membrane vesicle (OMV) purification and characterisation. a Purified form of OMVWT and OMVMel, isolated from parental bacteria by ultrafiltration and ultracentrifugation. b Dynamic light-scattering analysis of OMVs confirmed a particle size distribution in the range of 20 to $100 \mathrm{~nm}$. c Transmission electron micrograph showing the nano-sized $(<100 \mathrm{~nm})$, bilayered, circular morphology of OMVWT and OMVMel. Scale bars, $100 \mathrm{~nm}$. d Mean optoacoustic intensity (coloured line) as a function of wavelength for OMVWT and OMVMel

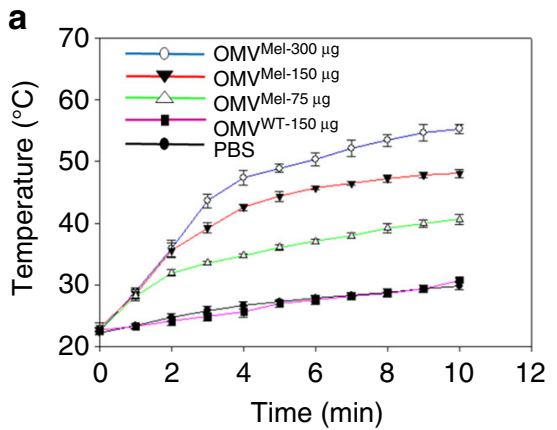

b
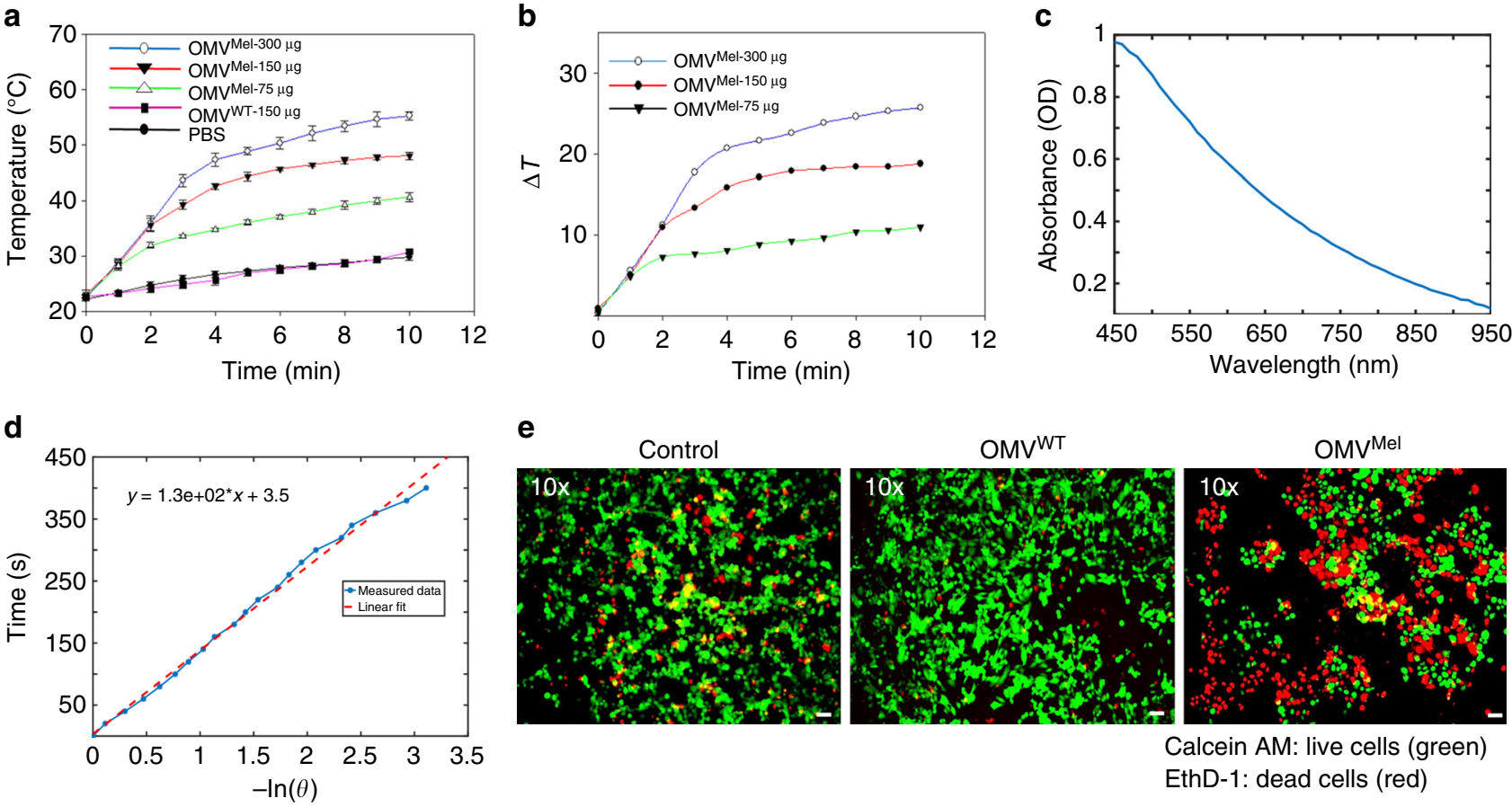

e

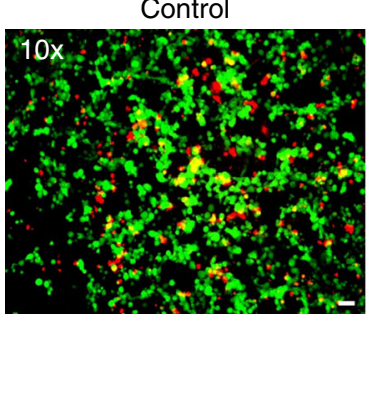

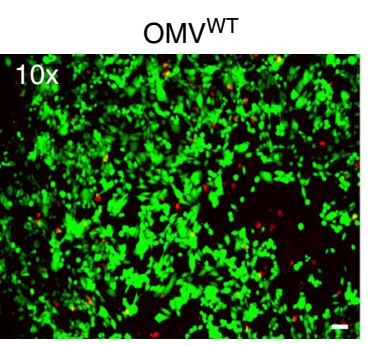

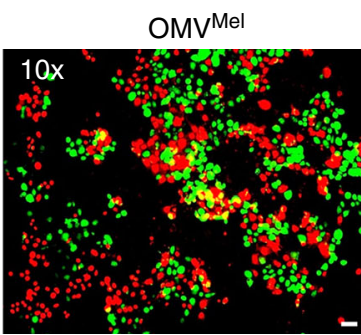

Calcein AM: live cells (green) EthD-1: dead cells (red)

Fig. 3 In vitro photothermal therapy using OMVMel. a Temperature curves of OMVMel, OMVWT and phosphate-buffered saline (PBS) during exposure to $750 \mathrm{~nm}$ light $\left(650 \mathrm{~mW} \mathrm{~cm}^{-2}\right)$ over a period of $10 \mathrm{~min}$. OMV, outer membrane vesicle. b Plot of temperature change $(\Delta T)$ over a period of $10 \mathrm{~min}$ as a function of OMVMel concentration. c Absorbance spectra of OMVMel ( 150 $\mu \mathrm{g})$ solution obtained using a spectrometer. $\mathbf{d}$ Plot of time as a function of - In $(\theta)$ for the raw data and a linear fit during cooling after $10 \mathrm{~min}$ of irradiation as described for a. e Fluorescence images of $4 \mathrm{~T} 1$ cells treated with PBS, OMVWT or OMVMel, then irradiated with a laser as described in the Methods. Viable cells were stained green with calcein-acetoxymethyl (AM), while dead cells were stained red with ethidium homodimer-1 (EthD-1). Scale bars, $20 \mu \mathrm{m}$ 
a
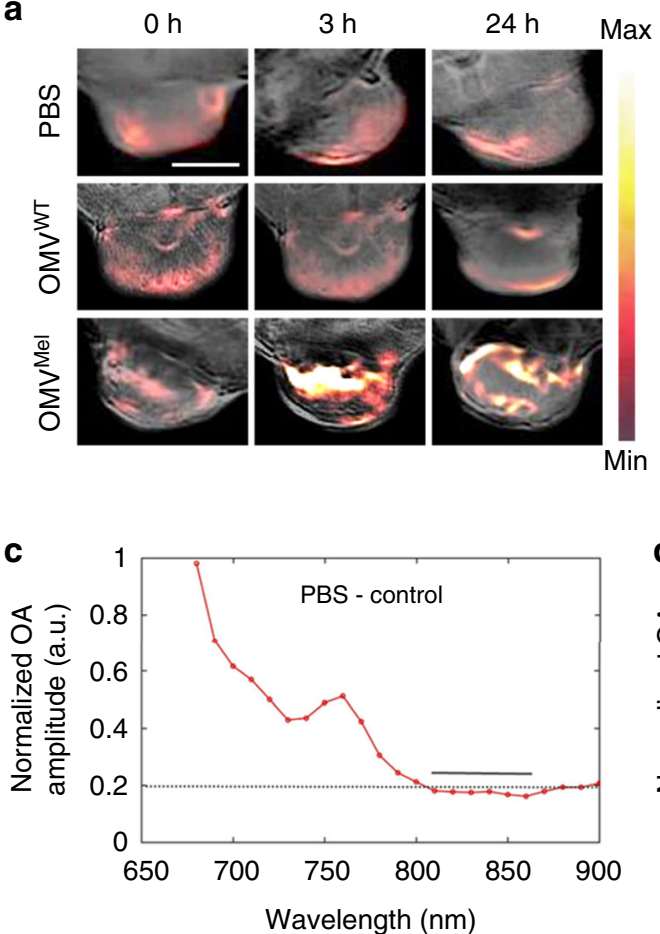

b

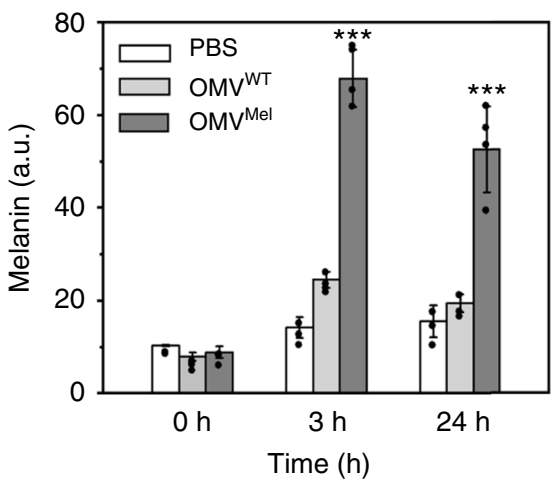

d

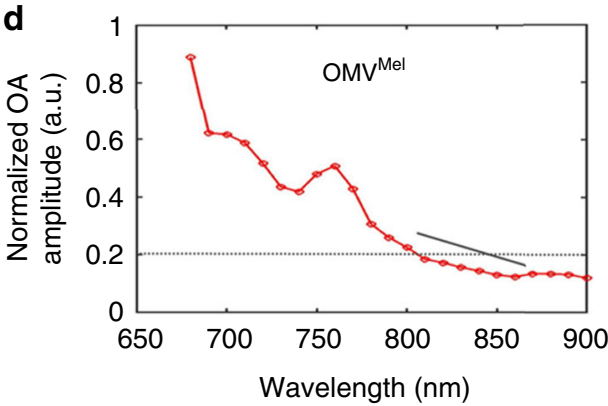

Fig. 4 In vivo multi-spectral optoacoustic tomography (MSOT) imaging. a 4T1 tumour-bearing mice were given a single injection of phosphate-buffered saline (PBS) $(n=3)$ or $150 \mu \mathrm{g}$ of OMVWT $(n=4)$ or OMVMel $(n=4)$ via the tail vein. Tumour-specific accumulation over time was monitored using a commercially available preclinical MSOT system. Scale bar, $4 \mathrm{~mm}$. OMV, outer membrane vesicle. $\mathbf{b}$ Melanin concentration in the tumour was measured over time. A single mean value was calculated over the tumour region. Mean values and error bars were expressed as mean \pm SD, inter-group differences were assessed for significance using the paired $t$-test compared to control and differences were considered significant if $\star_{\star \star \star} p<0.001$. $\mathbf{c}$, $\mathbf{d}$ Optoacoustic spectra from the tumour region of animals treated with $\mathbf{c}$ PBS or $\mathbf{d} O M V M e l$

animals treated intratumourally and exposed to the laser, most tumour tissue appeared necrotic and the tumour mass nearly disappeared; in animals treated intravenously, tumour growth was reduced by approximately $43 \%$ as shown in Fig. 5 b. No antitumour effects were observed in animals treated with PBS or in animals treated with OMVs in the absence of laser exposure. Throughout these phototherapy experiments, animals in all groups appeared normal and showed stable body weight (Fig. 5c), with no overt signs of toxicity. Our results suggest that OMV $\mathrm{Mel}$ can be used for in vivo optoacoustic imaging and phototherapy.

In vivo safety and immune responses. To confirm the in vivo safety of OMVs for photothermal therapy, we investigated whether single-dose systemic injection of PBS or OMVs $\left(\mathrm{OMV}^{\mathrm{WT}}\right.$, $\mathrm{OMV}^{\Delta \mathrm{msbB}}$ or $\mathrm{OMV}^{\mathrm{Mel}}$ ) would stimulate the immune system in C57BL/6 mice. The dose of OMVs was $75 \mu \mathrm{g}$, similar to the photothermal therapy experiment. Then, levels of the cytokines tumour necrosis factor- $\alpha$ (TNF- $\alpha$ ), interleukin-6 (IL-6) and interferon- $\gamma$ (IFN- $\gamma$ ) in serum were measured by enzyme-linked immunosorbent assay (ELISA). Cytokine levels were evaluated at 2 and $24 \mathrm{~h}$ in order to monitor early and delayed immune responses $^{16}$. All three types of OMVs increased serum levels of the three cytokines at $2 \mathrm{~h}$, with OMVWT triggering the greatest increases (Supplementary Figure 2a). In all cases, cytokine levels decreased close to baseline by $24 \mathrm{~h}$. In addition, histology of heart, liver, spleen and kidney at $24 \mathrm{~h}$ after injection did not indicate significant organ damage under these treatment conditions (Supplementary Figure 2b). No animal mortality occurred in any of the groups. These results suggest that the underacylated LPS on $\mathrm{OMV}^{\triangle \mathrm{msbB}}$ and $\mathrm{OMV}^{\mathrm{Mel}}$ induce milder systemic inflammation than the intact LPS on OMVWT, and that the modified OMVs are well tolerated upon systemic administration.

\section{Discussion}

Here we describe melanin-containing OMVs as a biocompatible contrast agent that may allow longitudinal imaging of tumours in vivo using optoacoustics, which offers several advantages over fluorescence methods for cancer monitoring. Fluorescence imaging does not offer good spatial resolution deeper than $0.5 \mathrm{~mm}$ because tissue strongly scatters light ${ }^{40-43}$. Optoacoustics, in contrast, generates optical images by recording the acoustic waves, which tissue does not strongly scatter ${ }^{21,44}$. In addition to offering images of higher resolution from deeper-lying tissue, optoacoustics is better suited than fluorescence for theranostics. Fluorescent agents cannot optimally emit fluorescence and heat target tissue locally, because the two processes of fluorescence and nonradioactive decay compete with each other. In the case of optoacoustics, contrast agents can be optimised for photothermal conversion efficiency, such that they simultaneously give strong acoustic signal while also converting light energy to heat.

We showed with transmission electron microscopy that naturally produced OMVs was able to effectively package melanin expressed from a transgene. Melanin was presumably encapsulated into OMVs together with various cytosolic-, periplasmic- or membrane-bound components during vesicle generation and secretion, though the exact mechanism for melanin packaging is not known ${ }^{45,46}$. Importantly, the generated $\mathrm{OMV}^{\mathrm{Mel}}$ particles remained sub-micrometre in size, with intact circular morphology, which indeed is similar to previously reported bioengineered OMVs under study for cancer-specific drug delivery ${ }^{16}$. OMV $\mathrm{Mel}$ show good dispersion properties and small particle size, allowing them to remain in circulation for an extended time, which facilitates passive accumulation in the tumour tissue by the EPR effect. We performed these experiments in a bacterial strain with a mutated form of the $m s b B$ gene. The resulting under-acylation 

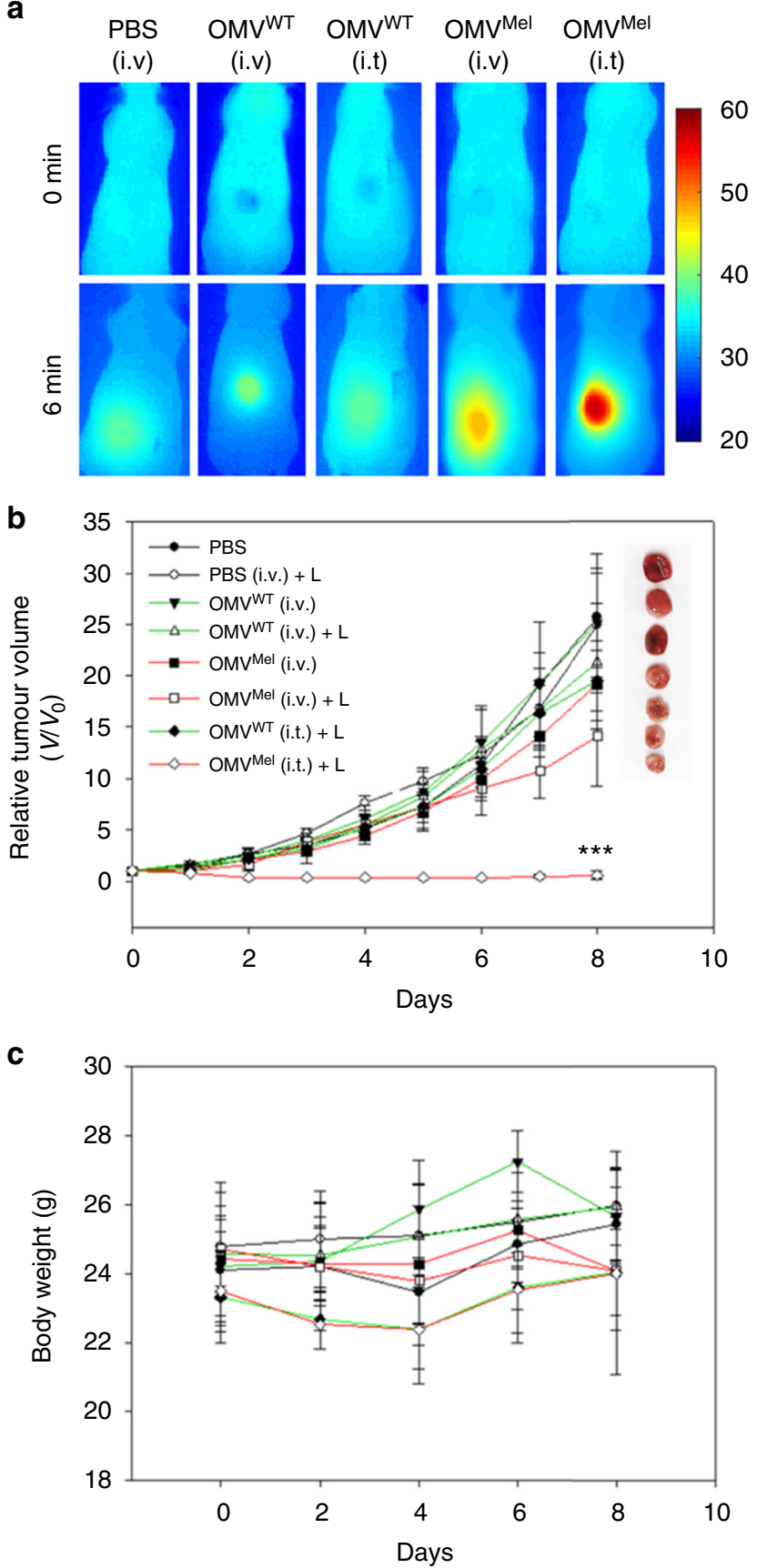

Fig. 5 In vivo photothermal therapy. a Infrared (IR) thermal images of 4T1 tumour-bearing mice before and after laser irradiation $\left(1.5 \mathrm{~W} \mathrm{~cm}^{-2}, 800 \mathrm{~nm}\right.$, $6 \mathrm{~min})$. Before irradiation, animals were injected with phosphate-buffered saline (PBS) intravenously or with OMVMel or OMVWT intravenously (i.v.) or intratumourally (i.t.). b Tumour growth curves. Representative images of dissected tumours are also shown, except for OMVMel (i.t.) + L, which had nearly disappeared. Mean values and error bars are presented as mean $\pm S D$, inter-group differences were assessed for significance using the paired $t$-test compared to control ( ${ }^{\star \star \star} p<0.001$ vs. PBS with laser treatment; $n=4$ ). c Body weight of all animals was recorded during each treatment, with all animals appearing healthy throughout the study based on eating and behaviour. OMV, outer membrane vesicle; $L$, laser irradiation

of lipid A substantially reduces the endotoxic activity of lipopolysaccharide, leading to weaker Toll-like receptor-dependent host immune reactions and increased in vivo safety 16,17,30,47. The same strain has previously been used to develop OMVs for in vivo drug delivery applications ${ }^{16}$. Using less endotoxic bacterial strains is attractive for facilitating translational research on bacterially derived particles ${ }^{16,17}$. Furthermore, the produced melanin was non-toxic to the bacterial cells. The entire process of melanin encapsulation is a natural event, so it does not require any sophisticated synthesis skills, and $\mathrm{OMV}^{\mathrm{Mel}}$ can easily be scaled up cost-effectively using well-established large-volume bacterial culture and purification techniques.

The absorption coefficient of OMVMel was found to be severalfold higher than that of tissue. As a result, OMVMel is expected to show strong optoacoustic signal. It appears that the proteolipid envelope of OMVs does not influence the absorption properties of encapsulated melanin biopolymers. In addition to NIR absorption, OMVMel show suitable properties for deep-tissue optoacoustic imaging and photothermal therapy. It offers a photothermal conversion efficiency of $18.6 \%$, and it allows local heating in a time- and melanin-dependent manner. Within $2 \mathrm{~min}$ of NIR laser irradiation, the temperature of $\mathrm{OMV}^{\mathrm{Mel}}$ suspensions increased beyond $45^{\circ} \mathrm{C}$, which is sufficient to kill cancer cells in vivo ${ }^{35-37,48}$. Since body temperature is around $37^{\circ} \mathrm{C}$, these results suggest that $\mathrm{OMV}^{\mathrm{Mel}}$ that has accumulated into tumours can easily be heated above $45^{\circ} \mathrm{C}$ upon NIR irradiation. When we irradiated $\mathrm{OMV}^{\mathrm{Mel}}$-treated $4 \mathrm{~T} 1$ cancer cells in culture, massive cytotoxic effects were observed that were consistent with good photothermal conversion efficiency. Next, we demonstrated the efficacy of photothermal therapy in vivo. Maximal temperature on the tumour surface reached 56 and $47^{\circ} \mathrm{C}$ after intratumoural or intravenous injection of OMV ${ }^{\mathrm{Mel}}$, compared to $39^{\circ} \mathrm{C}$ in PBStreated controls. Regardless of whether $\mathrm{OMV}^{\mathrm{Mel}}$ was delivered intratumourally or intravenously, it triggered obvious necrosis after laser exposure, and the tumour was nearly completely destroyed in animals treated intratumourally. These findings demonstrate the potential of $\mathrm{OMV}^{\mathrm{Mel}}$ as an effective nanoheater for diagnostic imaging and thermal therapy. Our in vivo photothermal therapy experiments were performed with continuous wave laser illumination. Our group previously demonstrated that photodynamic therapy can be performed with pulsed laser illumination ${ }^{29}$, and hence it should be possible to heat tumours with a nanosecond-pulsed laser illumination of MSOT as long as sufficient power can be delivered. In this case, MSOT could be used for image-guided therapy. Optoacoustic measurements can be used to estimate temperature distribution during photothermal therapy ${ }^{49}$, and hence an ideal theranostic system may combine phototherapy based on OMVMel with continuous monitoring of local temperature and $\mathrm{OMV}^{\mathrm{Mel}}$ distribution using MSOT.

Due to its natural origin, OMV ${ }^{\mathrm{Mel}}$ is a biocompatible and biodegradable agent that can be produced on a large scale in a cost-effective manner ${ }^{16,50-52}$. Here we explored its potential as a biologically derived theranostic agent. Our work has shown noninvasive monitoring of OMVMel using MSOT, and we demonstrated light-based heating with $\mathrm{OMV}^{\mathrm{Mel}}$ in vitro and in tumourbearing athymic nude mice. We used nude mice because their lack of pigmentation and skin hair facilitate optoacoustic analysis. However, this prevented us from determining whether OMV ${ }^{\text {Mel }}$ can stimulate immune responses against tumours. OMVs have been shown to induce the production of antitumour cytokines, including IFN- $\gamma$, in tumours of immunocompetent mice ${ }^{17}$. Repeated administration of OMVs can induce sustained production of IFN- $\gamma$ in tumour tissue as well as long-term immune memory ${ }^{17}$. Further detailed work is needed to examine whether, as suggested for other $\mathrm{OMVs}^{17}$, our $\mathrm{OMV}^{\mathrm{Mel}}$ can synergistically attack tumours by locally heating the tissue and triggering a longterm immune response to prevent cancer recurrence; indeed, hyperthermia can increase blood flow and immune cell infiltration. 
OMVs carry several proteins and LPS on membrane surface, which might trigger systemic toxicity ${ }^{16-20}$. In order to address safety issues of OMVs, we carried out a separate study using immunocompetent mice. OMVWT induced substantially stronger Toll-like receptor responses than $\mathrm{OMV}^{\Delta \mathrm{msbB}}$ and $\mathrm{OMV}^{\mathrm{Mel}}$ based on serum cytokine assays (Supplementary Figure 2a), reflecting that the latter two OMV types were prepared from a lowendotoxicity $E$. coli that is generally well tolerated. The OMV platform appears less likely to induce chronic systemic toxicity or other side effects, based on reports of bioengineered OMVs that were repeatedly injected into a mouse cancer model ${ }^{16,17}$. An important question is what happens to OMV ${ }^{\mathrm{Mel}}$ or melanin, which can function as a self-antigen. Studies with other OMVs suggest that they are degraded in late endosomes or lysosomes ${ }^{16,50,51}$, and that intracellular melanin is broken down by NADPH-dependent oxidoreductase ${ }^{53}$. The melanin in intact $\mathrm{OMV}^{\mathrm{Mel}}$ may remain hidden from the immune system because it is embedded within the bacterial membrane. Future work should verify and extend our findings by checking for systemic toxicity or toxic effects in major organs upon repeated administration of modified OMVs in small and large animals. Also, potential synergies between photothermal therapy mediated by melanin and immune responses triggered by the OMVs themselves should be explored. Long-term stability of $\mathrm{OMV}^{\mathrm{Mel}}$ upon standing should be evaluated, since this is important for clinical applications.

The efficacy of $\mathrm{OMV}^{\mathrm{Mel}}$ as a specific anti-cancer therapy depends on its ability to target tumours, and here we demonstrate such an ability in vivo. Optoacoustic signal due to $\mathrm{OMV}^{\mathrm{Mel}}$ was evident in tumours soon after intravenous injection, and this signal increased during $120 \mathrm{~min}$, suggesting tumour accumulation via the EPR effect. At the same time, appreciable melanin signal from $\mathrm{OMV}^{\mathrm{Mel}}$ was observed in a tumour-adjacent region just below the skin as well as in liver and kidney. This suggests that $\mathrm{OMV}^{\mathrm{Mel}}$ can persist in other organs, and the implications of this for safety should be explored further. It may not necessarily have toxic effects, since photothermal therapy usually involves shining activating light only on the tumour. The present work focused on imaging biodistribution of $\mathrm{OMV}^{\mathrm{Mel}}$ using MSOT. Future work should examine the fate of intact $\mathrm{OMV}^{\mathrm{Mel}}$ and the released melanin in vivo in greater detail.

Contrast enhancement in optoacoustic imaging originally relied on metal particles, in particular gold nanoparticles ${ }^{54,55}$. Although such particles can be produced with desired physical dimensions (size and shape) in a highly controlled way, they present several disadvantages for human use. They are difficult to reproduce on large scale and are vulnerable to photobleaching, which gives rise to variable optoacoustic response, and they can persist for long periods in tissue, increasing the risk of toxic effects ${ }^{54,55}$. We and others have shown that the use of dye-based nanoparticles absorbing in the NIR, such as quenched hexacene dye nanoparticles (referred as $\mathrm{QH}_{2}$ particles), can be a potent alternative to NIR metal nanoparticles for strong optoacoustic signal generation ${ }^{56}$. Importantly, $\mathrm{QH}_{2}$ particles can quench unwanted fluorescence and offer greater photostability in the NIR region than metal compounds. We also developed polymethine dyes such as DY-635 and DY-780 conjugated to polymeric scaffolds, and we showed that they could be used for organ-selective elimination, optoacoustic imaging and drug delivery; in fact, DY780 proved to be suitable for MSOT imaging ${ }^{57}$. The work herein takes the next step in generating bioengineered optoacoustic probes with the potential to seamlessly translate into clinical and commercial successes. Importantly, advances in biosynthesis and bioprocessing have provided scalable and robust platforms for manufacturing of such bioengineered theranostics to meet clinical needs.
MSOT operates in the NIR spectral region, where penetration of tissue to depths of several centimetres becomes possible because of low light attenuation, and laser exposure of the sample remains within the ANSI (American National Standards Institute) biosafety limits ${ }^{58}$. High molecular detection specificity is achieved by resolving (unmixing) multiple spectral signatures in tissues ${ }^{22}$. Clinical translation of MSOT is limited due to the lack of imaging probes that are biocompatible and scalable. Bioengineered OMVs or similar cell membrane-derived particles exhibit improved theranostic efficacy as well as reduced toxicity ${ }^{16,17,19}$. Preclinical success of such biological nanoparticles for diagnostic or therapeutic applications can have paradigm-shifting effects on clinical or commercial translation of biological agents.

Here we acquired MSOT data with a transducer array having 256 channels covering a $270^{\circ}$ field of view (in a two-dimensional plane). Light was delivered using a fibre bundle with six outputs, but high photothermal efficiency requires delivering light at a specific location of the body with a single output fibre. This can be achieved using handheld systems with single-point illumination or line illumination, but such systems feature limited detection angles, i.e., $145^{\circ}$ in a two-dimensional set-up, and $90^{\circ}$ in a three-dimensional set-up. As a result, quantification is challenging. Accurate quantification methods are being designed based on sparse recovery ${ }^{59}$, which may enable real-time threedimensional theranostic applications of OMVMel.

We report a bacterial membrane-derived nanoparticle system for optoacoustic imaging. The entirely biological design of this system may effectively avoid the long-term toxicity issues associated with many synthetic agents that deposit in various tissues and exhibit poor clearance. The ability to produce OMV ${ }^{\mathrm{Mel}}$ from bacteria means that the vesicles can be loaded with diverse genetically encoded agents suitable for optoacoustic imaging and image-guided therapy ${ }^{60}$. Potentially useful cargo proteins include fluorescent proteins, several optoacoustic-suitable chromoproteins and phytochromes ${ }^{61,62}$, anti-cancer toxins $s^{63}$ and peptides (e.g., aptides) $)^{64}$ or proteins (e.g., affibodies) $)^{14,16,65}$ that target specific tissues and therefore provide stronger therapeutic efficacy with weaker side effects. We anticipate that the OMVs presented in this work will be a starting point for future theranostic studies focused on simultaneous optoacoustic imaging and cancer therapeutics.

\section{Methods}

Plasmid construction, bacterial strain and growth. The melA gene of Rhizobium etli encoding tyrosinase (kindly provided by Professor Guillermo Gosset, Universidad Nacional Autónoma de México, Mexico) ${ }^{32}$ was cloned into pGEX-4T-1 (GE Healthcare, Freiburg, Germany). The construct pGEX-4T-1-melA was transformed into msbB mutant W3110-K12 E. coli (kindly provided by Professor Sangyong Jon, KAIST, South Korea ${ }^{16}$. Bacteria were cultured in 1.5-L flasks at $30^{\circ} \mathrm{C}$ and $180 \mathrm{rpm}$ for 5 days. Tyrosinase production was induced at OD 0.6 using isopropyl $\beta$-D-1-thiogalactopyranoside (IPTG) at a final concentration of $0.5 \mathrm{mM}$ To support melanin production the media were supplemented with $94.5 \mathrm{mg} \mathrm{CuSO}_{4}$ as well as $1 \mathrm{~g} \mathrm{~L}$-tyrosine. OMV ${ }^{\mathrm{Mel}}$ were produced in msbB mutant W3110-K12 E coli transformed with pGEX-4T-1-melA plasmid. OMVWT were produced from untransformed msbB mutant W3110-K12 E. coli.

OMV purification and characterisation. Based on our previous work ${ }^{16}$, OMVs were produced as follows. E. coli were cultured as described above. Next, bacteria cells were removed by centrifugation at $7500 \times g$ for $45 \mathrm{~min}$ at $4{ }^{\circ} \mathrm{C}$. The resulting supernatant was filtered by passing through a $0.45-\mu \mathrm{m}$ membrane filter (Nalgene, Thermo Scientific), and concentrated to $100 \mathrm{~mL}$ using $100-\mathrm{K}$ ultrafiltration membrane. The concentrate was further precipitated using ammonium sulphate (at final concentration, $400 \mathrm{~g} \mathrm{~L}^{-1}$ ) at $4{ }^{\circ} \mathrm{C}$ overnight. Crude OMVs were obtained by centrifugation at $12,000 \times g$ for $45 \mathrm{~min}$, the resulting pellet was resuspended in $1 \mathrm{~mL}$ PBS and was layered over a sucrose gradient $(1 \mathrm{~mL}$ each of $2.5,1.6$ and $0.6 \mathrm{M}$ sucrose) and separated from free melanin by ultracentrifugation at $150,000 \times g$ for $3-5 \mathrm{~h}$ at $4{ }^{\circ} \mathrm{C}$. The collected OMV fractions were washed with PBS with centrifugation at $150,000 \times g$ for $1-2 \mathrm{~h}$ at $4{ }^{\circ} \mathrm{C}$, resuspended in $1 \mathrm{~mL}$ PBS containing $15 \%$ glycerol, filtered through $0.45-\mu \mathrm{m}$ cellulose acetate filters and stored at $-20^{\circ} \mathrm{C}$ until use. In the case of OMV ${ }^{\mathrm{WT}}$, total protein concentration was estimated using 
the bicinchoninic acid (BCA) assay (Thermo Scientific), and this was defined to be the OMVWT concentration. Since black colour prevents protein estimation with the BCA assay, we had to develop an alternative procedure to determine OMV $\mathrm{OMl}^{\mathrm{Mel}}$ concentration. Concentrations of OMV ${ }^{\mathrm{Mel}}$ solutions were estimated based on the size of the pellet relative to the pellet obtained from OMVWT solutions (by ultracentrifugation) of known concentration. We verified the accuracy of this approach by comparing intensities of major protein bands obtained after fractionating $\mathrm{OMV}^{\mathrm{WT}}$ and $\mathrm{OMV}^{\mathrm{Mel}}$ on sodium dodecyl sulphate-polyacrylamide gel electrophoresis. OMV samples were characterised with respect to size and morphology using an electrophoretic light-scattering apparatus (Malvern Zetasizer) and transmission electron microscope (Zeiss Libra 120 Plus, Carl Zeiss NTS $\mathrm{GmbH}$, Oberkochen, Germany).

Cell culture and in vivo tumour experiments. 4T1 mammary gland carcinoma (CRL-2539, ATCC, Manassas, VA, USA) was cultured in RPMI-1640 medium supplemented with $10 \%$ foetal bovine serum and antibiotics. Cells were incubated at $37^{\circ} \mathrm{C}$ in $5 \% \mathrm{CO}_{2}$. Animal procedures were reviewed and approved by the Animal Care and Handling Office of Helmholtz Zentrum München and by the Government of Upper Bavaria. Sample sizes were chosen based on guidance from the literature. Investigators were not blinded to the identity of groups. Female athymic Fox-N-1 nude mice 6 weeks old (Envigo, Germany) were implanted subcutaneously with $4 \mathrm{~T} 1$ murine breast cancer cells $\left(0.8 \times 10^{6}\right.$ cells per animal). Tumour volume, calculated as (width) ${ }^{2} \times($ length $) \times 1 / 2$, and relative tumour volume, calculated as $V / V_{0}$ (where $V_{0}$ was the tumour volume when the treatment was initiated), was monitored at regular intervals and was expressed as group mean \pm standard deviation (SD). Tumour growth inhibition (TGI) was determined on the final day as \% TGI: $100 \% \times\left(T_{\mathrm{vol}}^{\mathrm{PBS}}-T_{\mathrm{vol}}^{\mathrm{OMV}}\right) \times\left(T_{\mathrm{vol}}^{\mathrm{PBS}}\right)^{-1}$, where $T_{\mathrm{vol}}$ is final tumour volume - initial tumour volume.

MSOT set-up and data acquisition. Phantom and mice data were acquired using a commercially available MSOT scanner (MSOT256-TF, iThera Medical GmbH, Munich, Germany) ${ }^{66}$. Nanosecond-pulsed light was generated from a tuneable optical parametric oscillator (OPO) laser and delivered to the sample through a ring-type fibre bundle. The wavelength used for imaging was from 680 to $900 \mathrm{~nm}$ with a step size of $10 \mathrm{~nm}$ and 10 averages. Light is absorbed by the sample and generates an acoustic signal that propagates through the sample and is detected outside the sample. In our experiments, acoustic signals were detected as timeseries pressure readouts at 2030 discrete time points at 40 Mega samples per second using a cylindrically focused, 256-element transducer. The transducer array had a central frequency of $5 \mathrm{MHz}(-6 \mathrm{~dB}$ was approximately $90 \%$ ) with a radius of curvature of $40 \mathrm{~mm}$ and an angular coverage of $270^{\circ}$.

Acoustic data were measured using a transducer array with 256 elements and $270^{\circ}$ coverage at multiple laser wavelengths. The acquired acoustic data were filtered using a Chebyshev filter with cut-off frequencies of 0.1-7 MHz.

Optoacoustic images were reconstructed using a Tikhonov regularisation-based scheme using filtered optoacoustic data; the regularisation parameter was chosen automatically using L-curve methods ${ }^{67}$. The Tikhonov method was implemented using the least-squares QR method to reconstruct an image at each wavelength ${ }^{59}$. These spectral optoacoustic images were used to unmix melanin chromophores through a linear unmixing method that assumed that fluence did not influence unmixing. Melanin quantification was then performed on the unmixed melanin image from the tumour region by plotting mean intensity from a defined region of interest (corresponding to a similar tissue area in all animals) over time. Differences in mean melanin levels were considered significant if $p<0.01$. Advanced unmixing methods are being developed to quantify biomolecules ${ }^{68}$, but these methods have been extensively validated only for oxy- and deoxyhaemoglobin. Efforts are underway to extend these methods to other biomolecules such as melanin and lipids.

MSOT imaging of phantoms. A cylindrical agar phantom ${ }^{69}$ was prepared with two cylindrical holes, one for OMV ${ }^{\mathrm{WT}}$ and another for OMVMel. The cylindrical phantom contained $1.3 \%(\mathrm{w} / \mathrm{w})$ agar (Sigma-Aldrich, St. Louis, MO, USA) to provide solidity and $6 \%(\mathrm{v} / \mathrm{v})$ intralipid emulsion (20\%, Sigma-Aldrich) for light diffusion to enable uniform illumination of the holes. The added intralipid gives a reduced scattering coefficient of $10 \mathrm{~cm}^{-1}$, mimicking scattering in tissue ${ }^{33}$. The two cylindrical holes were $3 \mathrm{~mm}$ in diameter. MSOT data were acquired as described above to allow transversal plane imaging at a single position, located approximately in the middle of the phantom.

Measurement of photothermal activity. Photothermal activity was measured by placing PBS or OMVWT $\left(150 \mu \mathrm{g} \mathrm{mL}^{-1}\right)$ or OMV Mel solution $\left(75,150\right.$ or $300 \mu \mathrm{g} \mathrm{mL}^{-1}$ equivalent concentration) in a quartz cuvette, and then exposing solutions to an NIR light source at $750 \mathrm{~nm}$ (Tunable Optical Parametric Oscillator Laser, InnoLas Laser, Krailling, Germany) while monitoring temperature changes with a digital thermometer for up to $10 \mathrm{~min}$. Concentration-response experiments were performed at a fixed laser power $\left(650 \mathrm{~mW} \mathrm{~cm}^{-2}\right)$ and compared with PBS as a control.
Cytotoxicity study. Fluorescence-based live/dead cell assays were carried out using the molecular probes calcein-acetoxymethyl (AM) and ethidium homodimer-1 (Invitrogen). Briefly, cells were seeded into 96-well plates and incubated with or without $\mathrm{OMV}^{\mathrm{WT}}$ or OMV ${ }^{\mathrm{Mel}}$ for $6 \mathrm{~h}$. After the media were replaced with fresh media, cells were exposed to nanosecond-pulsed laser treatment $(730-830 \mathrm{~nm}$, $650 \mathrm{~mW} \mathrm{~cm}^{-2}$ ) for $6 \mathrm{~min}$ and then stained with calcein-AM and ethidium homodimer-1 following the manufacturer's instructions. Cells were examined under a fluorescence microscope to determine numbers of live and dead cells (Thermo Scientific).

MSOT imaging of mice and estimation of melanin. Animal procedures were reviewed and approved by the Animal Care and Handling Office of Helmholtz Zentrum München and by the Government of Upper Bavaria. To examine in vivo cancer targeting, mice bearing $4 \mathrm{~T} 1$ tumours $\left(\sim 150 \mathrm{~mm}^{3}\right)$ were given a single injection of either PBS, OMV WT $(\sim 150 \mu \mathrm{g})$ or an equivalent amount of OMVMel via the tail vein ( $n=4$ for OMV treatment and $n=3$ for PBS treatment). An additional mouse was scanned to monitor distribution of OMV $\mathrm{OMl}^{\mathrm{Mel}}$ in tumour, liver and kidney from 0 to $120 \mathrm{~min}$ after intravenous injection of $\mathrm{OMV}^{\mathrm{Mel}}$ (mouse was killed immediately after imaging). Mice were anaesthetised with $1.8 \%$ isoflurane in oxygen, which was delivered throughout MSOT data acquisition. Ultrasound gel was applied to the mice before acquisition to enable coupling between the tissue and water medium. Tissue homing and retention were monitored at $3 \mathrm{~h}$ and $24 \mathrm{~h}$ post injection with MSOT. In a separate experiment, OMV Mel $(\sim 75 \mu \mathrm{g})$ was injected via the tail vein, and then the tumour and nearby region (just below the skin) were monitored with MSOT continuously for $120 \mathrm{~min}$ without moving the mouse (mouse was killed immediately after imaging). Melanin intensities are averages calculated from 6 adjacent slices from the tumour centre. Sample sizes for animal studies were chosen based on institutional recommendations with guidance from the literature. Investigators were not blinded to animal group allocations.

In vivo photothermal therapy. Tumour volume and body weight were recorded at regular intervals. Once the tumour volume reached approximately $100 \mathrm{~mm}^{3}$, mice were divided into eight groups ( $n=4$ mice/group). The groups were treated with PBS intravenously, with or without laser treatment; with $\mathrm{OMV}^{\mathrm{WT}}$ or OMVMel (each $\sim 75 \mu \mathrm{g}$ ) intravenously, with or without laser treatment; and with OMVWT or $\mathrm{OMV}^{\mathrm{Mel}}$ intratumourally, with laser treatment. At $3 \mathrm{~h}$ after injection, tumours were irradiated with a continuous wave laser $\left(1.5 \mathrm{~W} \mathrm{~cm}^{-2}, 800 \mathrm{~nm}\right)$ for $6 \mathrm{~min}$. Temperature at tumour sites was recorded immediately before and after laser irradiation using an IR thermal camera (FLIR i60). Investigators were not blinded to the identity of groups.

In vivo safety and immune responses. The safety and immune responses to OMVs were assessed in female C57BL/6 mice 6 weeks old. Animals were divided into four groups ( $n=5$ mice/group): (1) vehicle control (PBS), (2) OMVWT, (3) $\mathrm{OMV}^{\triangle \mathrm{msbB}}$ and (4) OMV $\mathrm{OMl}^{\mathrm{Mel}}$. OMVs were administered intravenously, then blood was collected via cardiac puncture (under surgical anaesthesia) at 2 and $24 \mathrm{~h}$. Levels of the TNF- $\alpha$, IL- 6 and IFN- $\gamma$ in serum were quantified at $450 \mathrm{~nm}$ using commercial ELISA kits (R\&D Systems) according to the manufacturer's instructions. Vital organs including heart, liver, spleen and kidney were extracted, fixed and frozen in OCT embedding gel. Tissue blocks were sectioned to $10 \mu \mathrm{m}$, and stained with haematoxylin and eosin. Images were obtained by light microscopy (Carl Zeiss). Investigators were not blinded to the identity of the groups.

Statistics. Sample sizes were chosen based on guidance from the literature. Animals of the same gender, age and genetic background were randomised for grouping. Statistical analyses were performed using SPSS 18.0 (IBM, Chicago, IL, USA). Inter-group differences were assessed for significance using the paired $t$-test. Results were expressed as mean $\pm \mathrm{SD}$, and differences were considered significant if $p<0.01$.

\section{Data availability}

All data presented in the paper are available from the authors upon reasonable request.

Received: 23 March 2018 Accepted: 7 February 2019

Published online: 07 March 2019

\section{References}

1. Shanmugam, V., Selvakumar, S. \& Yeh, C. S. Near-infrared light-responsive nanomaterials in cancer therapeutics. Chem. Soc. Rev. 43, 6254-6287 (2014).

2. Xie, J., Lee, S. \& Chen, X. Nanoparticle-based theranostic agents. Adv. Drug Deliv. Rev. 62, 1064-1079 (2010).

3. Michalet, X. et al. Quantum dots for live cells, in vivo imaging, and diagnostics. Science 307, 538-544 (2005). 
4. Nam, J. et al. Chemo-photothermal therapy combination elicits anti-tumor immunity against advanced metastatic cancer. Nat. Commun. 9, 1074 (2018).

5. Mukherjee, S. et al. Potential theranostics application of bio-synthesized silver nanoparticles (4-in-1 system). Theranostics 4, 316-335 (2014).

6. Tian, Q. et al. Hydrophilic flower-like CuS superstructures as an efficient 980 $\mathrm{nm}$ laser-driven photothermal agent for ablation of cancer cells. Adv. Mater. 23, 3542-3547 (2011).

7. Zha, Z. B., Yue, X. L., Ren, Q. S. \& Dai, Z. F. Uniform polypyrrole nanoparticles with high photothermal conversion efficiency for photothermal ablation of cancer cells. Adv. Mater. 25, 777-782 (2013).

8. Miao, W. et al. Photo-decomposable organic nanoparticles for combined tumor optical imaging and multiple phototherapies. Theranostics $\mathbf{6}$, 2367-2379 (2016).

9. Peer, D. et al. Nanocarriers as an emerging platform for cancer therapy. Nat. Nanotechnol. 2, 751-760 (2007).

10. Desai, N. Challenges in development of nanoparticle-based therapeutics. AAPS J. 14, 282-295 (2012).

11. Yoo, J. W., Irvine, D. J., Discher, D. E. \& Mitragotri, S. Bio-inspired, bioengineered and biomimetic drug delivery carriers. Nat. Rev. Drug. Discov. 10, 521-535 (2011).

12. Alvarez-Erviti, L. et al. Delivery of siRNA to the mouse brain by systemic injection of targeted exosomes. Nat. Biotechnol. 29, 341-345 (2011).

13. $\mathrm{Hu}, \mathrm{C} . \mathrm{M}$. J. Erythrocyte membrane-camouflaged polymeric nanoparticles as a biomimetic delivery platform. Proc. Natl. Acad. Sci. USA 108, 10980-10985 (2011).

14. Gujrati, V. et al. Bioengineered yeast-derived vacuoles with enhanced tissuepenetrating ability for targeted cancer therapy. Proc. Natl. Acad. Sci. USA 113, 710-715 (2016).

15. MacDiarmid, J. A. et al. Bacterially derived $400 \mathrm{~nm}$ particles for encapsulation and cancer cell targeting of chemotherapeutics. Cancer Cell 11, 431-445 (2007).

16. Gujrati, V. et al. Bioengineered bacterial outer membrane vesicles as cellspecific drug-delivery vehicles for cancer therapy. ACS Nano 8, 1525-1537 (2014).

17. Kim, O. Y. et al. Bacterial outer membrane vesicles suppress tumor by interferon-gamma-mediated antitumor response. Nat. Commun. 8, 626 (2017).

18. Gerritzen, M. J. H., Martens, D. E., Wijffels, R. H., van der Pol, L. \& Stork, M. Bioengineering bacterial outer membrane vesicles as vaccine platform. Biotechnol. Adv. 35, 565-574 (2017).

19. Chen, Q., Rozovsky, S. \& Chen, W. Engineering multi-functional bacterial outer membrane vesicles as modular nanodevices for biosensing and bioimaging. Chem. Commun. (Camb.). 53, 7569-7572 (2017).

20. Acevedo, R. et al. Bacterial outer membrane vesicles and vaccine applications. Front. Immunol. 5, 121 (2014).

21. Ntziachristos, V. \& Razansky, D. Molecular imaging by means of multispectral optoacoustic tomography (MSOT). Chem. Rev. 110, 2783-2794 (2010).

22. Ntziachristos, V. Going deeper than microscopy: the optical imaging frontier in biology. Nat. Methods 7, 603-614 (2010).

23. Tomaszewski, M. R. et al. Oxygen enhanced optoacoustic tomography (OEOT) reveals vascular dynamics in murine models of prostate cancer. Theranostics 7, 2900-2913 (2017).

24. McNally, L. R. et al. Current and emerging clinical applications of multispectral optoacoustic tomography (MSOT) in oncology. Clin. Cancer Res. 22, 3432-3439 (2016).

25. Simon, J. D. Spectroscopic and dynamic studies of the epidermal chromophores trans-urocanic acid and eumelanin. ACC Chem. Res. 33 307-313 (2000).

26. Stritzker, J. et al. Vaccinia virus-mediated melanin production allows MR and optoacoustic deep tissue imaging and laser-induced thermotherapy of cancer. Proc. Natl. Acad. Sci. USA 110, 3316-3320 (2013).

27. Fan, Q. et al. Transferring biomarker into molecular probe: melanin nanoparticle as a naturally active platform for multimodality imaging. J. Am. Chem. Soc. 136, 15185-15194 (2014).

28. Liu, Y. et al. Dopamine-melanin colloidal nanospheres: an efficient nearinfrared photothermal therapeutic agent for in vivo cancer therapy. Adv. Mater. 25, 1353-1359 (2013).

29. Neuschmelting, V. et al. WST11 vascular targeted photodynamic therapy effect monitoring by multispectral optoacoustic tomography (MSOT) in mice. Theranostics 8, 723-734 (2018).

30. Kim, S. H. et al. Structural modifications of outer membrane vesicles to reine them as vaccine delivery vehicles. Biochim. Biophys. Acta 1788, 2150-2159 (2009).

31. Somerville, J. E. Jr., Cassiano, L. \& Darveau, R. P. Escherichia coli msbB gene as a virulence factor and a therapeutic target. Infect. Immun. 67, 6583-6590 (1999).

32. Cabrera-Valladares, N. et al. Expression of the melA gene from Rhizobium etli CFN42 in Escherichia coli and characterization of the encoded tyrosinase. Enzym. Microb. Tech. 38, 772-779 (2006).
33. Jacques, S. L. Optical properties of biological tissues: a review (vol 58, pg R37, 2013). Phys. Med. Biol. 58, 5007-5008 (2013).

34. Kim, M. et al. Thermohydrogel containing melanin for photothermal cancer therapy. Macromol. Biosci. 17, 1-6 (2017).

35. Hildebrandt, B. et al. The cellular and molecular basis of hyperthermia. Crit. Rev. Oncol. Hematol. 43, 33-56 (2002).

36. Falk, M. H. \& Issels, R. D. Hyperthermia in oncology. Int. J. Hyperth. 17, 1-18 (2001).

37. Dewey, W. C. Arrhenius relationships from the molecule and cell to the clinic. Int. J. Hyperth. 25, 3-20 (2009).

38. Rai, P. et al. Development and applications of photo-triggered theranostic agents. Adv. Drug Deliv. Rev. 62, 1094-1124 (2010).

39. Zou, L. et al. Current approaches of photothermal therapy in treating cancer metastasis with nanotherapeutics. Theranostics 6, 762-772 (2016).

40. Acedo, P., Stockert, J. C., Canete, M. \& Villanueva, A. Two combined photosensitizers: a goal for more effective photodynamic therapy of cancer. Cell Death Dis. 5, e1122 (2014).

41. Ma, X. P. et al. Assessment of asthmatic inflammation using hybrid fluorescence molecular tomography-x-ray computed tomography. J. Biomed. Opt. 21, 15009 (2016).

42. Ma, X. et al. Integrin-targeted hybrid fluorescence molecular tomography/Xray computed tomography for imaging tumor progression and early response in non-small cell lung cancer. Neoplasia 19, 8-16 (2017).

43. Glinzer, A. et al. Targeting elastase for molecular imaging of early atherosclerotic lesions. Arterioscler. Thromb. Vasc. Biol. 37, 525-533 (2017).

44. Wang, L. V. \& Hu, S. Photoacoustic tomography: in vivo imaging from organelles to organs. Science 335, 1458-1462 (2012).

45. Bonnington, K. E. \& Kuehn, M. J. Protein selection and export via outer membrane vesicles. Biochim. Biophys. Acta 1843, 1612-1619 (2014).

46. Jan, A. T. Outer membrane vesicles (OMVs) of Gram-negative bacteria: a perspective update. Front. Microbiol. 8, 1053 (2017).

47. Hajjar, A. M., Ernst, R. K., Tsai, J. H., Wilson, C. B. \& Miller, S. I. Human Toll-like receptor 4 recognizes host-specific LPS modifications. Nat. Immunol. 3, 354-359 (2002)

48. Dewey, W. C. Arrhenius relationships from the molecule and cell to the clinic. Int. J. Hyperth. 10, 457-483 (1994).

49. Landa, F. J. O., Dean-Ben, X. L., Sroka, R. \& Razansky, D. Volumetric optoacoustic temperature mapping in photothermal therapy. Sci. Rep. 7, 9695 (2017).

50. Amano, A., Takeuchi, H. \& Furuta, N. Outer membrane vesicles function as offensive weapons in host-parasite interactions. Microbes Infect. 12, 791-798 (2010).

51. Furuta, N. et al. Porphyromonas gingivalis outer membrane vesicles enter human epithelial cells via an endocytic pathway and are sorted to lysosomal compartments. Infect. Immun. 77, 4187-4196 (2009).

52. Ninh, C., Cramer, M. \& Bettinger, C. J. Photoresponsive hydrogel networks using melanin nanoparticle photothermal sensitizers. Biomater. Sci. 2, 766-774 (2014).

53. Borovansky, J. \& Elleder, M. Melanosome degradation: fact or fiction. Pigment Cell Res. 16, 280-286 (2003).

54. Gujrati, V., Mishra, A. \& Ntziachristos, V. Molecular imaging probes for multi-spectral optoacoustic tomography. Chem. Commun. (Camb.). 53, 4653-4672 (2017)

55. Nie, L. \& Chen, X. Structural and functional photoacoustic molecular tomography aided by emerging contrast agents. Chem. Soc. Rev. $\mathbf{4 3}$ 7132-7170 (2014).

56. Nunes, A. et al. Quenched hexacene optoacoustic nanoparticles. J. Mater. Chem. B 6, 44-55 (2018).

57. Press, A. T. et al. Cell type-specific delivery of short interfering RNAs by dyefunctionalised theranostic nanoparticles. Nat. Commun. 5, 5565 (2014).

58. LIA. American National Standard for Safe Use of Lasers. ANSI Z136.1 (Laser Institute of America, Orlando, Florida, 2014).

59. Han, Y. et al. Three-dimensional optoacoustic reconstruction using fast sparse representation. Opt. Lett. 42, 979-982 (2017).

60. Liu, C. et al. Advances in imaging techniques and genetically encoded probes for photoacoustic imaging. Theranostics 6, 2414-2430 (2016).

61. Li, Y. et al. Engineering dark chromoprotein reporters for photoacoustic microscopy and FRET imaging. Sci. Rep. 6, 22129 (2016).

62. Mark, J. et al. Photoacoustic imaging of fluorophores using pump-probe excitation. Biomed. Opt. Express 6, 2522-2535 (2015).

63. Patyar, S. et al. Bacteria in cancer therapy: a novel experimental strategy. J. Biomed. Sci. 17, 21 (2010)

64. Kim, S. et al. Bio-inspired design and potential biomedical applications of a novel class of high-affinity peptides. Angew. Chem. Int. Ed. Engl. 51, 1890-1894 (2012)

65. Orlova, A. et al. Tumor imaging using a picomolar affinity HER2 binding affibody molecule. Cancer Res. 66, 4339-4348 (2006). 
66. Razansky, D., Buehler, A. \& Ntziachristos, V. Volumetric real-time multispectral optoacoustic tomography of biomarkers. Nat. Protoc. 6 1121-1129 (2011).

67. Shaw, C. B., Prakash, J., Pramanik, M. \& Yalavarthy, P. K. Least squares QRbased decomposition provides an efficient way of computing optimal regularization parameter in photoacoustic tomography. J. Biomed. Opt. 18, 80501 (2013)

68. Tzoumas, S. et al. Eigenspectra optoacoustic tomography achieves quantitative blood oxygenation imaging deep in tissues. Nat. Commun. 7, 12121 (2016)

69. Beziere, N. et al. Dynamic imaging of PEGylated indocyanine green (ICG) liposomes within the tumor microenvironment using multi-spectral optoacoustic tomography (MSOT). Biomaterials 37, 415-424 (2015).

\section{Acknowledgements}

This project has received funding from the European Research Council (ERC) under the European Union's Horizon 2020 research and innovation programme under grant agreement no. 694968 (PREMSOT). The research leading to these results was supported by the Deutsche Forschungsgemeinschaft (DFG), Germany (Gottfried Wilhelm Leibniz Prize 2013, NT 3/10-1) as well as by the DFG as part of the CRC 1123 (Z1). J.P. acknowledges support from the Alexander von Humboldt Postdoctoral Fellowship Program. We wish to thank Professor Guillermo Gosset (Universidad Nacional Autónoma de México) for providing the plasmid encoding R. etli tyrosinase, Professor Sangyong Jon (Korea Advanced Institute of Science and Technology, South Korea) for providing msbB mutant W3110-K12 E. coli, Andreas Schroeppel and Dr. Otmar Schmid (Comprehensive Pneumology Center Munich, Institute of Lung Biology and Disease, Helmholtz Zentrum München) for helping us perform particle size measurements in their laboratory, Dr. Juan Antonio Aguilar-Pimentel (German Mouse Clinic/Institute of Experimental Genetics, Helmholtz Zentrum München) for providing the IR thermal camera, and Kanuj Mishra, Nian Liu, Dr. Doris Bengel, Ruth Hillermann, Sarah Glasl and Pia Anzenhofer for assisting with experimental procedures. We also wish to thank Dr. A. Chapin Rodríguez for helpful suggestions on the manuscript.

\section{Author contributions}

V.G. conceived the OMV ${ }^{\mathrm{Mel}}$ system and designed the study. V.G. and J.P. performed the experiments, J.P. processed the MSOT data and helped analyse the data. J.M.-N. assisted with in vivo experiments and MSOT data analysis. A.S. subcloned the melA gene and assisted with bacterial culture. U.K. assisted with in vivo experiments and animal maintenance. G.M. and V.G. performed transmission electron microscopy, which M.A and A.W. supervised. V.N. provided significant intellectual input, helped interpret the results and supervised the research. All authors contributed to writing the paper.

\section{Additional information}

Supplementary Information accompanies this paper at https://doi.org/10.1038/s41467019-09034-y.

Competing interests: V.N. is a shareholder in iThera Medical GmbH, Munich, Germany. The remaining authors declare no competing interests.

Reprints and permission information is available online at http://npg.nature.com/ reprintsandpermissions/

Journal peer review information: Nature Communications thanks Peter van der Ley and the other anonymous reviewer(s) for their contribution to the peer review of this work. Peer reviewer reports are available.

Publisher's note: Springer Nature remains neutral with regard to jurisdictional claims in published maps and institutional affiliations.

\begin{abstract}
cc) (i) Open Access This article is licensed under a Creative Commons Attribution 4.0 International License, which permits use, sharing, adaptation, distribution and reproduction in any medium or format, as long as you give appropriate credit to the original author(s) and the source, provide a link to the Creative Commons license, and indicate if changes were made. The images or other third party material in this article are included in the article's Creative Commons license, unless indicated otherwise in a credit line to the material. If material is not included in the article's Creative Commons license and your intended use is not permitted by statutory regulation or exceeds the permitted use, you will need to obtain permission directly from the copyright holder. To view a copy of this license, visit http://creativecommons.org/ licenses/by/4.0/.
\end{abstract}

(C) The Author(s) 2019 